\title{
Anisotropic magnetic excitations and incipient Néel order in $\mathrm{Ba}\left(\mathrm{Fe}_{1-x} \mathrm{Mn}_{x}\right)_{2} \mathrm{As}_{2}$
}

\author{
Fernando A. Garcia, ${ }^{1, *}$ Oleh Ivashko, ${ }^{2}$ Daniel E. McNally,${ }^{3}$ Lakshmi Das, ${ }^{2}$ Mario M. Piva, ${ }^{4}$ C. Adriano, ${ }^{4}$ \\ Pascoal G. Pagliuso, ${ }^{4}$ Johan Chang, ${ }^{2}$ Thorsten Schmitt, ${ }^{3}$ and Claude Monney ${ }^{5}$ \\ ${ }^{1}$ Instituto de Física, Universidade de São Paulo, São Paulo-SP, 05508-090, Brazil \\ ${ }^{2}$ Physik-Institut, Universitaet Zuerich, Winterthurerstrasse 190, 8057 Zurich, Switzerland \\ ${ }^{3}$ Photon Science Division, Swiss Light Source, Paul Scherrer Institut, 5232 Villigen PSI, Switzerland \\ ${ }^{4}$ Instituto de Física "Gleb Wataghin”, UNICAMP, 13083-859, Campinas, SP, Brazil \\ ${ }^{5}$ Physics Department, University of Fribourg, Ch. du Musée 3, 1700 Fribourg, Switzerland
}

(Received 25 September 2018; revised manuscript received 24 January 2019; published 14 March 2019)

\begin{abstract}
It is currently understood that high temperature superconductivity (SC) in the transition metal $(M)$ substituted iron arsenides $\mathrm{Ba}\left(\mathrm{Fe}_{1-x} M_{x}\right)_{2} \mathrm{As}_{2}$ is promoted by magnetic excitations with wave vectors $(\pi, 0)$ or $(0, \pi)$. It is known that while a small amount of Co substitution lead to SC, the same does not occur for Mn for any value of $x$. In this work, magnetic excitations in the iron arsenides $\mathrm{Ba}\left(\mathrm{Fe}_{1-x} \mathrm{Mn}_{x}\right)_{2} \mathrm{As}_{2}(x=0.0,0.007,0.009,0.08)$ are investigated by means of resonant inelastic x-ray scattering (RIXS) at the Fe $L_{3}$ edge, for momentum transfer $\boldsymbol{q}$ along the high symmetry Brillouin zone $(\pi, 0)$ and $(\pi, \pi)$ directions. It is shown that with increasing Mn content $(x)$, the excitations become anisotropic both in dispersion and lineshape. Both effects are detected even for small values of $x$, evidencing a cooperative phenomenon between the Mn impurities, that we ascribe to emerging Néel order of the Mn spins. Moreover, for $x=0.08$, the excitations along $\boldsymbol{q} \|(\pi, 0)$ are strongly damped and nearly nondispersive. This result suggests that phases of arsenides containing local moments at the FeAs layers, as in $\mathrm{Mn}$ or Cr substituted phases, do not support high temperature SC due to the absence of the appropriate magnetic excitations.
\end{abstract}

DOI: 10.1103/PhysRevB.99.115118

\section{INTRODUCTION}

Iron based superconductors [1] encompass a broad family of electronic correlated materials for which orbital and spin excitations are believed to play a key role in determining the system properties [2]. Of special interest are the iron arsenides, of which $\mathrm{BaFe}_{2} \mathrm{As}_{2}$ is particularly well explored and the subject of much attention $[3,4]$.

$\mathrm{BaFe}_{2} \mathrm{As}_{2}$ (BFA) undergoes an itinerant spin density wave (SDW) phase transition at about $T_{N}=134 \mathrm{~K}$ [5]. For BFA, superconductivity (SC) is achieved by means of chemical substitution on either $\mathrm{Ba}, \mathrm{Fe}$, or As sites, which suppresses the SDW phase [4]. SC is observed with critical temperatures $\left(T_{S C}\right)$ as high as $\sim 20-30 \mathrm{~K}$ in the case of transition metal substitution at the Fe site $[3,4]$.

It is known that the composition versus temperature ( $x$ vs $T$ ) phase diagram of the transition metal $(T M)$ substituted $\mathrm{Ba}\left(\mathrm{Fe}_{1-x} T M_{x}\right)_{2} \mathrm{As}_{2}$ systems presents an unexpected asymmetry, which concerns the fact that $\mathrm{SC}$ is not observed for $\mathrm{Cr}$ and $\mathrm{Mn}$ substituted samples, that are on the hole-doped side of the phase diagram [6]. The unique aspect related to $\mathrm{Mn}$, or $\mathrm{Cr}$ substitution, is the presence of strongly localized magnetic moments at the dopant site [7-9], contrasting with the moments observed for $\mathrm{Co}$ and $\mathrm{K}$ substituted phases, which still present a high degree of itinerancy [10]. Indeed, magnetic moments within the FeAs layers seem to be detrimental to

*Corresponding author: fgarcia@if.usp.br
$\mathrm{SC}$, which in other cases has been shown to persist when magnetism is formed between these planes [11,12].

The present work discusses magnetic excitations of $\mathrm{Ba}\left(\mathrm{Fe}_{1-x} \mathrm{Mn}_{x}\right)_{2} \mathrm{As}_{2}(x=0.0,0.007,0.009,0.08)$ as probed by means of resonant inelastic x-ray scattering (RIXS), for momentum transfer $\boldsymbol{q}$ along the high symmetry Brillouin zone (BZ) $(\pi, 0)$ and $(\pi, \pi)$ directions. Cooper pairing in the high temperature superconducting phases in arsenides, likely a $s \pm$ (extended $s$-wave) phase, is understood to be promoted by excitations with $(\pi, 0) /(0, \pi)$ wave vectors, derived from the $\mathrm{Fe}$ electronic degrees of freedom [13,14]. Excitations with a $(\pi, \pi)$ wave vector would give rise to a relatively weaker superconducting phase, with a gap of $d$-wave symmetry [13-15]. Our experiment reveals that with increasing Mn content, the Fe derived magnetic excitations become anisotropic, both in dispersion and lineshape. In particular, the excitations along the $(\pi, 0)$ direction are strongly damped and nearly nondispersive. Therefore, it is found that the system lacks the magnetic excitations that promote the most efficient channel for interorbital SC pairing [14]. Thus, our findings contribute to understanding the absence of SC in phases of arsenides containing $\mathrm{Mn}$, in particular, or in phases containing $\mathrm{Cr}$, that also introduces strongly localized moments at the FeAs layers. In addition, the $\mathrm{Fe}$ derived excitations are affected by small amounts of Mn, evidencing a cooperative behavior of the $\mathrm{Mn}$ impurities, that is ascribed to an emerging Néel order in the system.

The case of Mn substitution presents a particular interesting case study $[6,16-18]$. The observed behavior is involved and understood to be related to the presence of strong $Q_{\text {Néel }}=$ 
$(\pi, \pi)$ short range spin fluctuations [17,18]. The decisive role of magnetic moments sitting in the Mn sites is clear since, as suggested by x-ray absorption (XAS) and photoemission spectroscopy (PES), the Mn ions do not act as charge dopants [19].

\section{METHODS}

Our experiments were performed at the ADRESS beamline of the Swiss Light Source at Paul Scherrer Institute [20,21]. All RIXS spectra shown in this work have been acquired for an incident photon energy tuned to the maximum of the Fe $L_{3}$ - XAS edge and for $\pi$-polarized incident photons. Each spectrum intensity has been normalized to the maximum intensity of the fluorescence peak $I_{0}$. The total energy resolution of the RIXS experiment was about $95 \mathrm{meV}$. The temperature was kept constant throughout the experiment at $T=15 \mathrm{~K}$ and a base pressure of $2 \times 10^{-10} \mathrm{mbar}$ or better was achieved. The 1-Fe magnetic (orthorhombic) unit cell is adopted here for describing the orientation of the samples [22]. $\mathrm{Ba}\left(\mathrm{Fe}_{1-x} \mathrm{Mn}_{x}\right)_{2} \mathrm{As}_{2}(x=0.0,0.007,0.009,0.08)$ single crystals were synthesized by the In-flux method as described in Ref. [23]. The resistivity of the samples was measured by employing a commercial physical properties measurements system (PPMS) from Quantum Design.

\section{RESULTS AND DISCUSSION}

In Figs. 1(a) and 1(b) $x$ vs $T$ phase diagrams for the Mn and Co substituted samples are presented (data from Ref. [6]). The red arrows and red squares indicate [Fig. 1(a)] the doping values at which samples were investigated in our experiments. The transition temperatures were determined by the peaks (or inflection points) on the resistivity curves, as it is shown in Fig. 1(c). By assuming that the SDW transition temperature is a qualitative measure of the energy scale of the magnetic interactions between the $\mathrm{Fe}$ derived itinerant spins in both systems, our Mn-substituted $x=0.08$ sample and the $x \sim$ 0.03 Co-substituted system are to be compared. As can be seen in Figs. 1(a) and 1(b), the systems have similar SDW onset, yet only the Co-substituted compound is a SC-SDW coexisting system.

Figures 1(d) and 1(e) display, respectively, XAS and RIXS spectra at the $\mathrm{Fe} L_{3}$ edge of our pristine $\mathrm{BaFe}_{2} \mathrm{As}_{2}$ samples. The XAS spectrum obtained with $\pi$-polarized incident photons is in good agreement with previously reported results of $\mathrm{BaFe}_{2} \mathrm{As}_{2}$ [24,25]. The RIXS spectrum in Fig. 1(e) presents the main features observed for a diverse range of compositions of iron arsenides: a broad fluorescence line, reflecting the de-excitation of electrons from the valence bands having a high density of states of $\mathrm{Fe}$ character, and the magnetic excitation at low energy loss $\Delta E$. The latter is well defined and can be distinguished from the elastic peak at $\Delta E=0$. In general, RIXS experiments of arsenides unveiled dispersive magnetic excitations that were found to have nearly isotropic dispersions along the high symmetry $\mathrm{BZ}$ directions $(\pi, 0)$ and $(\pi, \pi)$, presenting characteristics that depend weakly on composition [25-28]. Contrasting with this picture, the RIXS spectra presented in this study display a different behavior as a function of composition.
In Figs. 2(a)-2(p) we show a representative survey of our results, focusing the attention on the elastic line and background subtraction, along with the magnetic excitation fitting. The figures show data of the $x=0.0$ and $x=0.08$ samples, for distinct $\|\boldsymbol{q}\|$ along the main directions of the BZ zone as indicated. For the parent compound, even at low $\|\boldsymbol{q}\|$ [Figs. 2(d) and 2(1)], the magnetic excitation can be distinguished from the elastic peak at $\Delta E=0$. Substitution by $\mathrm{Mn}$, however, unavoidably introduces scattering centers which increase the elastic line relative intensity, as observed for all spectra obtained for the $x=0.08$ sample. Indeed, the elastic lines of this sample overlap with the magnetic excitations. For $\boldsymbol{q}$ along $(\pi, \pi)$, the magnetic excitations are well defined for the highest values of $\|\boldsymbol{q}\|$ and appear as weak bumps for the lowest values of $\|\boldsymbol{q}\|$. For $\boldsymbol{q}$ along $(\pi, 0)$, even for the highest value of $\|\boldsymbol{q}\|$ the magnetic excitations overlap with the elastic line, a situation which calls for a careful procedure for its subtraction.

We extract the elastic line contribution assuming that the RIXS signal for $\Delta E>0$ is exclusively due to the elastic line and that it is symmetric with respect to $\Delta E=0$ [29]. We then mirror the data for $\Delta E>0$ and subtract it from the respective RIXS signal, unveiling the magnetic excitations along with the fluorescence background.
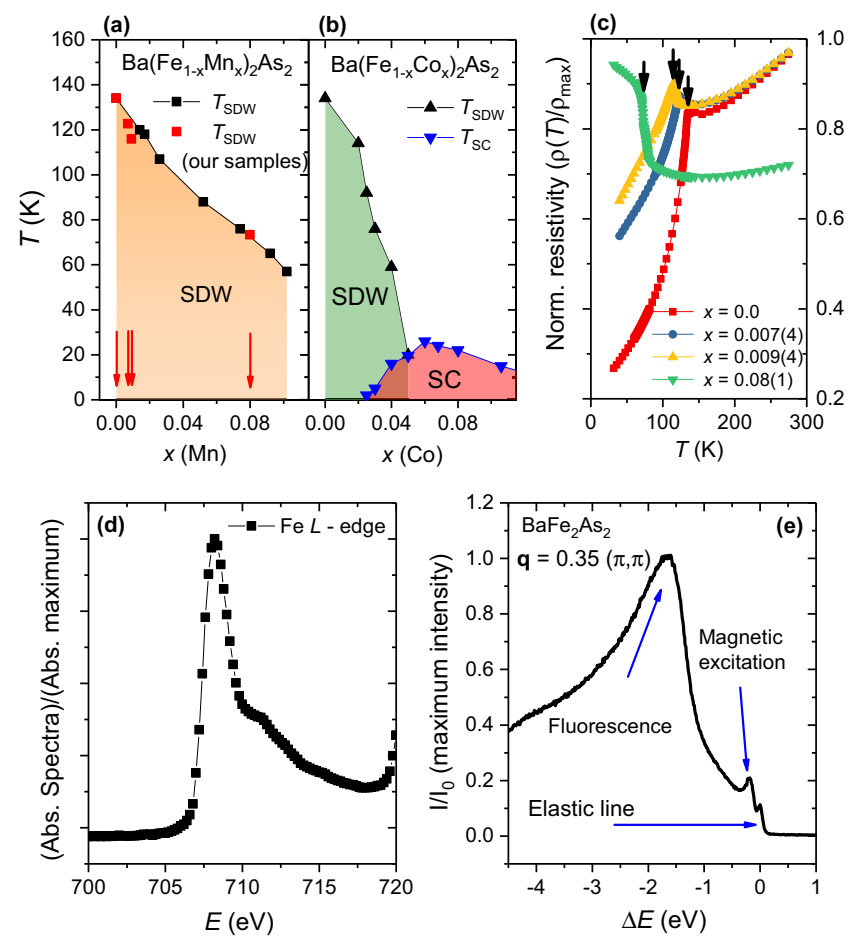

FIG. 1. $x$ vs $T$ phase diagram for the (a) $\mathrm{Ba}\left(\mathrm{Fe}_{1-x} \mathrm{Mn}_{x}\right)_{2} \mathrm{As}_{2}$ and (b) $\mathrm{Ba}\left(\mathrm{Fe}_{1-x} \mathrm{Co}_{x}\right)_{2} \mathrm{As}_{2}$ transition metal substituted iron arsenides (adapted from Ref. [6]). Red arrows in (a) point to the compositions investigated in our experiments. The transition temperatures were determined by the peaks, or inflections, of the resistivity measurements as shown in (c). (d) A typical XAS spectrum for the Fe $L_{3}$ edge is presented. (e) A representative RIXS spectrum is shown, wherein the main features of the RIXS spectra collected in our experiments are pointed out. 

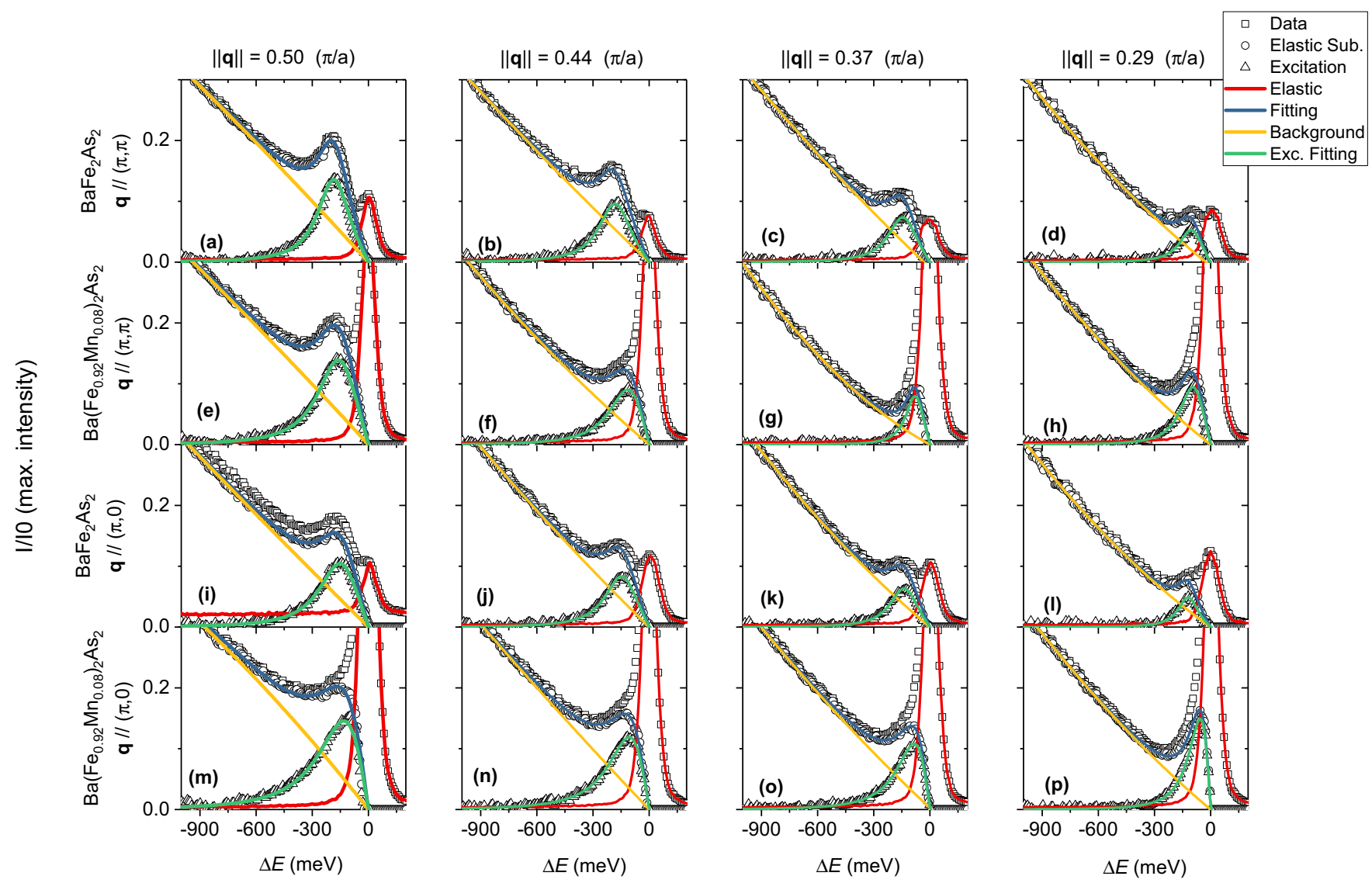

FIG. 2. Survey of representative RIXS spectra and analysis for the $x=0.0$ sample measured for $\boldsymbol{q}$ along the (a) $-(\mathrm{d})(\pi, \pi)$ and (i) $-(1)$ ( $\pi$, 0 ) directions, respectively, and for the $x=0.08$ sample with $\boldsymbol{q}$ along the (e)-(h) $(\pi, \pi)$ and (m)-(p) $(\pi, 0)$ directions, respectively. Open symbols denote the experimental data (squares), the elastic line subtracted data (circles), and the magnon excitations obtained after the background subtraction (triangles). The thick lines are fits of the spectra.

At low energy loss, the latter can be well described by a polynomial fitting [see yellow line in Figs. 2(a)-2(p)]. The RIXS magnetic excitation is proportional to the complex part of the dynamic susceptibility $\chi^{\prime \prime}(\omega)$ that is described by a damped harmonic oscillator. The associated expression reads:

$$
\begin{aligned}
\chi^{\prime \prime}(\omega) & =\chi_{0}^{\prime \prime}\left(\frac{\Gamma \omega}{\left(\omega^{2}-\omega_{0}^{2}\right)^{2}+\omega^{2} \Gamma^{2}}\right) \\
& =\frac{\chi_{0}^{\prime \prime}}{\omega_{\boldsymbol{q}}}\left(\frac{\Gamma / 2}{\left(\omega-\omega_{\boldsymbol{q}}\right)^{2}+(\Gamma / 2)^{2}}-\frac{\Gamma / 2}{\left(\omega+\omega_{\boldsymbol{q}}\right)^{2}+(\Gamma / 2)^{2}}\right),
\end{aligned}
$$

where $\chi_{0}^{\prime \prime}$ is a constant, $\omega_{0}$ is the excitation bare frequency (without the damping effect), $\Gamma$ is the excitation lifetime, and $\omega_{\boldsymbol{q}}=\sqrt{\omega_{0}^{2}-(\Gamma / 2)^{2}}$ is the propagation frequency of the excitation along the $\boldsymbol{q}$ direction. The final expression to fit the excitation is given by $\chi^{\prime \prime}(\omega)\left(1+n_{B}(\omega)\right)$ where $n_{B}(\omega)$ is the Bose-Einstein distribution function $\left(n_{B}=\left(\exp \left(\hbar \omega / k_{\mathrm{B}} T\right)-\right.\right.$ $1)^{-1}$ ). All the spectra of the parent compound are well described by the model outlined above and the results are in good agreement with what was found previously [25]. For the $x=0.08$ sample, all excitations exhibit large values of $\Gamma$, being strongly damped. For $\boldsymbol{q}$ along $(\pi, 0)$, in particular, the magnetic excitations are well spread in energy rendering
$\Gamma \approx \omega_{0}$ which, in turn, suggests that the excitations along $(\pi, 0)$ are heavily damped or even overdamped for the $x=$ 0.08 sample.

A more detailed inspection of the obtained spectra is presented Figs. 3(a)-3(d), wherein the magnetic excitations for the $\mathrm{Ba}\left(\mathrm{Fe}_{1-x} \mathrm{Mn}_{x}\right)_{2} \mathrm{As}_{2}$ samples at the two extreme dopings used here, $x=0.0$ and $x=0.08$, are presented. The excitations were measured at maximum accessible (by RIXS) momentum transfer $\|\boldsymbol{q}\|$, for $\boldsymbol{q}$ along the high symmetry BZ $(\pi, 0)$ and $(\pi, \pi)$ directions. The respective raw spectra with the elastic-line fits are shown in the insets.

The contrast between the results for our samples at the two extreme dopings, $x=0.0$ and $x=0.08$, is clear. For the parent compound, the fit magnon excitation frequencies $\omega_{q}$ along both directions are about $\omega_{q} \approx 170 \mathrm{meV}$, with the excitation along $(\pi, 0)$ being a little broader. In both cases, the proposed model fits well to the experimental lineshape. For the $x=0.08$ sample, one observes that for $\boldsymbol{q}$ along the $(\pi, \pi)$ direction the excitation peaks at $\approx-170 \mathrm{meV}$ and the fit frequency is $\omega_{(\pi, \pi)} \approx 100(10) \mathrm{meV}$. As for $\boldsymbol{q}$ along the $(\pi, 0)$ direction, the excitation peaks at about $-100 \mathrm{meV}$ and the excitation frequency is $\omega_{(\pi, 0)} \approx 60(15) \mathrm{meV}$ [30]. It is noteworthy that for both directions the excitation is considerably broader and there appears to be some extra spectral weight (indicated by the arrows) close to $\Delta E=0$ that is not properly described by the fitting function. For $\boldsymbol{q}$ along $(\pi, 0)$, this feature overlaps 


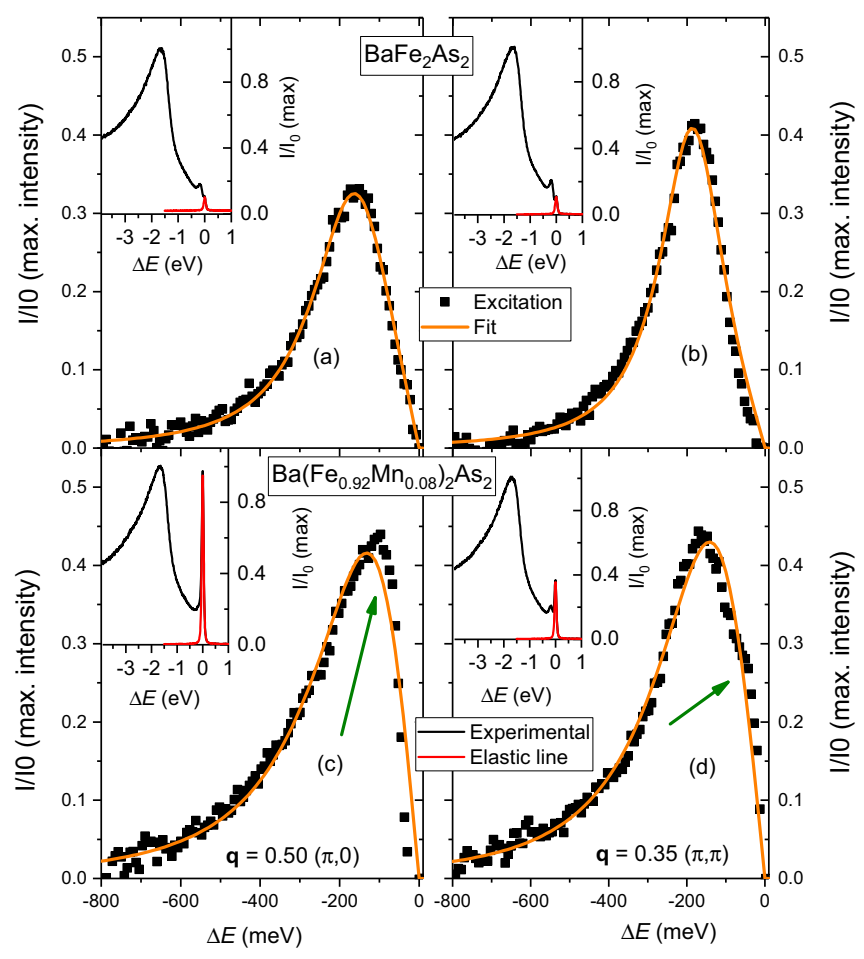

FIG. 3. Excitations for the $x=0.0$ sample with $\boldsymbol{q}$ along the (a) $(\pi, 0)$ and (b) $(\pi, \pi)$ directions, respectively, and excitations for the $x=0.08$ sample with $\boldsymbol{q}$ along the (c) $(\pi, 0)$ and (d) $(\pi, \pi)$ directions, respectively. The thick lines are fits of the spectra to a damped harmonic oscillator. The arrows in panels (c) and (d) point to features in the lineshape not captured by our model. The insets show the respective spectra before the removal of both the elastic line and background.

strongly with the main excitation. Since for $\boldsymbol{q}$ along $(\pi, \pi)$ the main excitation disperses a little further, this extra component can be better distinguished.

These results are surprising since the magnetic excitations observed by RIXS in doped iron arsenides did not show so far any considerable evolution with composition [25-27], similarly to what has been found for hole-doped cuprates [31,32]. Thus, anisotropic and composition dependent magnetic excitations are observed for iron arsenides by RIXS. Moreover, the excitations are not properly described by a simple model of a damped harmonic oscillator, which is unable to capture a very low energy feature close to $\Delta E=0$, the intensity of which is increasing with Mn content.

Therefore, to analyze the spectra obtained for other values of momentum transfer, we adopt a phenomenological description, taking the peak position at the maximum intensity, $\Delta E_{\max }$, of the low-energy excitation as a measurement of the excitation propagation energy. The spectra and peak positions are shown in Figs. 4(a) and 4(b) for the $x=0.0$ and $x=0.08$ samples as a function of momentum transfer $\|\boldsymbol{q}\|$ for the two directions being considered $[\boldsymbol{q}$ along $(\pi, 0)$, left panel and $\boldsymbol{q}$ along $(\pi, \pi)$, right panel]. Direct observation reveals that while for $\boldsymbol{q}$ along $(\pi, \pi)$ the spectra of both samples present a similar pattern as a function of $\|\boldsymbol{q}\|$, for $\boldsymbol{q}$ along $(\pi, 0)$ the excitation of the $x=0.08$ sample is nearly nondispersive. The scenario drawn on the basis of Figs. 4(a) and 4(b) is

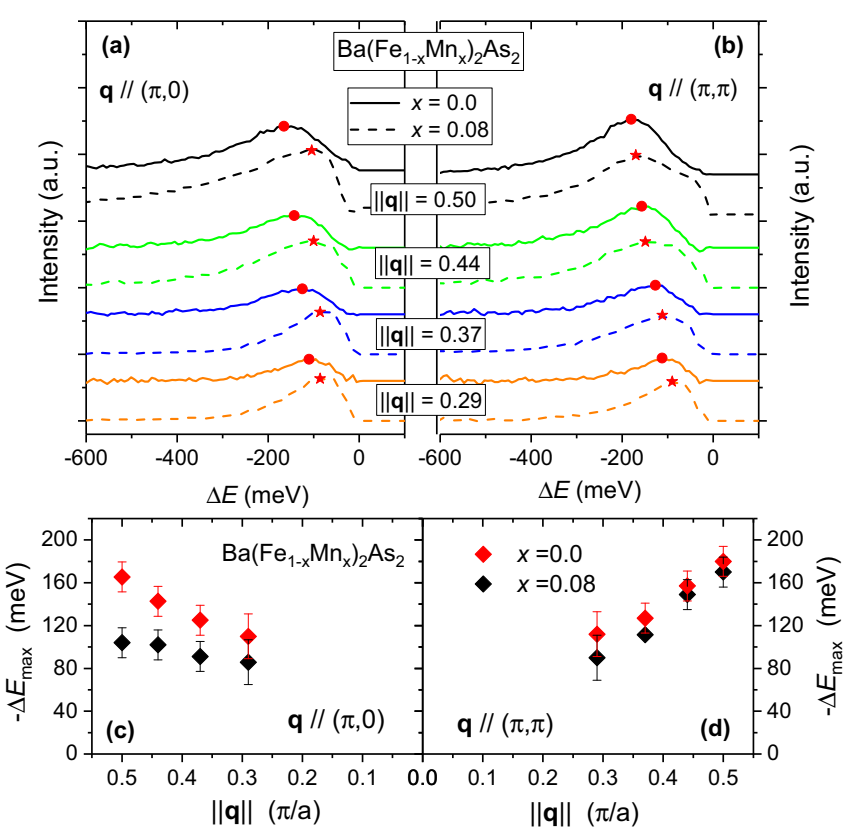

FIG. 4. Magnetic excitations for momentum transfer $\boldsymbol{q}$ (a) along the $(\pi, 0)$ direction and (b) along the $(\pi, \pi)$ direction $(x=0.0$ and $x=0.08$ samples) after removal of the fluorescence and elastic contributions, as a function of momentum transfer $\|\boldsymbol{q}\|$. Red dots and red stars mark the peak positions for the $x=0.0$ and $x=0.08$ samples, respectively. Energy loss (peak position), $-\Delta E_{\max }$, of the magnetic excitations as a function of $\|\boldsymbol{q}\|$ for (c) $\boldsymbol{q}$ along the $(\pi, 0)$ direction and (d) $\boldsymbol{q}$ along the $(\pi, \pi)$ direction.

summarized in Figs. 4(c) and 4(d), where the peak positions are marked by red diamonds and black diamonds for the two cases, respectively. The anisotropy of the excitation dispersion for the $x=0.08$ sample is clear: While the low-energy excitation disperses up to $170 \mathrm{meV}$ along $(\pi, \pi)$ in the $x=0.08$ sample, it hardly disperses from 80 to $100 \mathrm{meV}$ along $(\pi, 0)$.

The observed anisotropy between the excitations along $(\pi, 0)$ and $(\pi, \pi)$ in Mn-doped iron arsenides, both in their dispersions and lineshape, calls for an explanation. We start by discussing two possible mechanisms addressing the specifics of the RIXS physics in the arsenides. In short, these two mechanisms are (i) the increasing contribution of electronhole excitations at low-energy, overlapping with a possibly weakened magnetic excitation, or (ii) a magnetic excitation overdamped by strong scattering with intralayer magnetic impurities.

(i) RIXS at the Fe $L_{3}$ edge probes both Fe derived spin and charge excitations and both can in principle contribute to the low-energy excitations close to the elastic line. It has been shown that electron-hole excitations between bands close to the Fermi level can lead to dispersive peaks in RIXS, for instance in the semimetal $\mathrm{TiSe}_{2}$ [33], in the nickelate $\mathrm{NdNiO}_{3}$ [34] or in hole-doped cuprates [35,36]. In the case of doped iron arsenides, doping-induced changes of the electron bands at the BZ border could allow more electron-hole excitations between bands near the Fermi level. This would correspond to an increase of RIXS signal coming from electron-hole excitations upon doping along $(\pi, 0)$, since it is the vector connecting electron and hole pockets at $(\pi, 0)$ and at $\Gamma$ in iron arsenides. In the case of bands crossing the Fermi level, 
one would expect to see in RIXS electron-hole contributions already at zero energy loss. Thus, the small RIXS signal at very low energy (see green arrows in Fig. 3) could be due to some electron-hole excitations near the Fermi level. However, it is rather weak and could be due to some imperfect subtraction of the large elastic line at high $x$. We note that XAS and PES suggest that the Mn ions do not act as charge dopants [19], with the Mn $3 d$ partial density of states distributed over an energy range $2-13$ eV below the Fermi level.

(ii) A recent RIXS study of Eu-based iron arsenides has shown that the magnetic excitations are mostly unaffected by the presence of strong $\mathrm{Eu}^{2+} S=7 / 2$ local moments [27], evidencing that the unique feature related to Mn substitution is the appearance of strongly localized moments at the $\mathrm{Mn}$ sites inside the $\left(\mathrm{Fe}_{1-x} \mathrm{Mn}_{x}\right)_{2} \mathrm{As}_{2}$ layers. The Mn impurities introduce strong $Q_{\text {Néel }}=(\pi, \pi)$ short range spin fluctuations in the system $[17,18]$, which might have an indirect influence on the related magnetic excitations.

Indeed, here our main finding concerns the strong damping and the softening of the magnetic excitation along the $(\pi, 0)$ direction. In the ordered SDW phase of pnictides, it is interpreted that the magnons have predominant intraorbital $x y \rightarrow x y$ character [37]. Orbitals with $x y$ character contribute to bands whose extrema are centered around the BZ points $(\pi, 0)$ and $(0, \pi)$, which are separated by a $(\pi, \pi)$ wave vector $[14,38]$. Therefore, $Q_{\text {Néel }}=(\pi, \pi)$ fluctuations are able to scatter electrons with $x y$ character from distinct bands into one another, providing a possible mechanism for understanding why the excitations along the $(\pi, 0)$ direction are strongly affected by Mn substitution. We propose that $Q_{\text {Néel }}=(\pi, \pi)$ fluctuations lead to a transient change of the occupation number of the electronic states of $x y$ orbital character at $(\pi, 0)$ and $(0, \pi)$. We caution, however, that RIXS at the Fe $L_{3}$ edge can reach in-plane momentum transfers $\boldsymbol{q}$ up to $\sim 0.5(\pi, 0)$, meaning that we only probe a fraction of the magnon dispersion.

We now turn our attention to the relevance of our results to the physics of the arsenides. We first call attention that one must keep in mind that RIXS at the $\mathrm{Fe} L_{3}$ edge probes $\mathrm{Fe}$ derived excitations. Therefore, it is interesting that while $\mathrm{K}$ and Co substitution lead both to SC, the RIXS probed excitations are largely unaffected by the change of composition in these instances $[10,25,26]$. In contrast, SC is not observed for phases with Mn, that is here shown to be a strong scatter of the Fe derived excitations along the $(\pi, 0) /(0, \pi)$ directions. Thus, the absence of superconductivity in pnictide samples containing Mn spins can be understood in terms of electron scattering by $Q_{\text {Néel }}=(\pi, \pi)$ fluctuations which suppress the high temperature superconducting $s^{+-}$phase, favoring the more fragile $d$-wave superconductivity [15].

The specifics of Mn substitution should also be addressed. Indeed, the remarkable fact that small amounts of $\mathrm{Mn}$ impurities impact strongly the Fe derived magnetic excitations was not yet discussed. This finding suggests the kind of cooperative behavior of the Mn impurities as discussed in Ref. [39] or a strong coupling between the order parameter of the incipient Néel-type and the SDW ordering wave vector $Q_{\mathrm{SDW}}=(\pi, 0)$ or $(0, \pi)$ as proposed in Ref. [40]. Such collective behavior
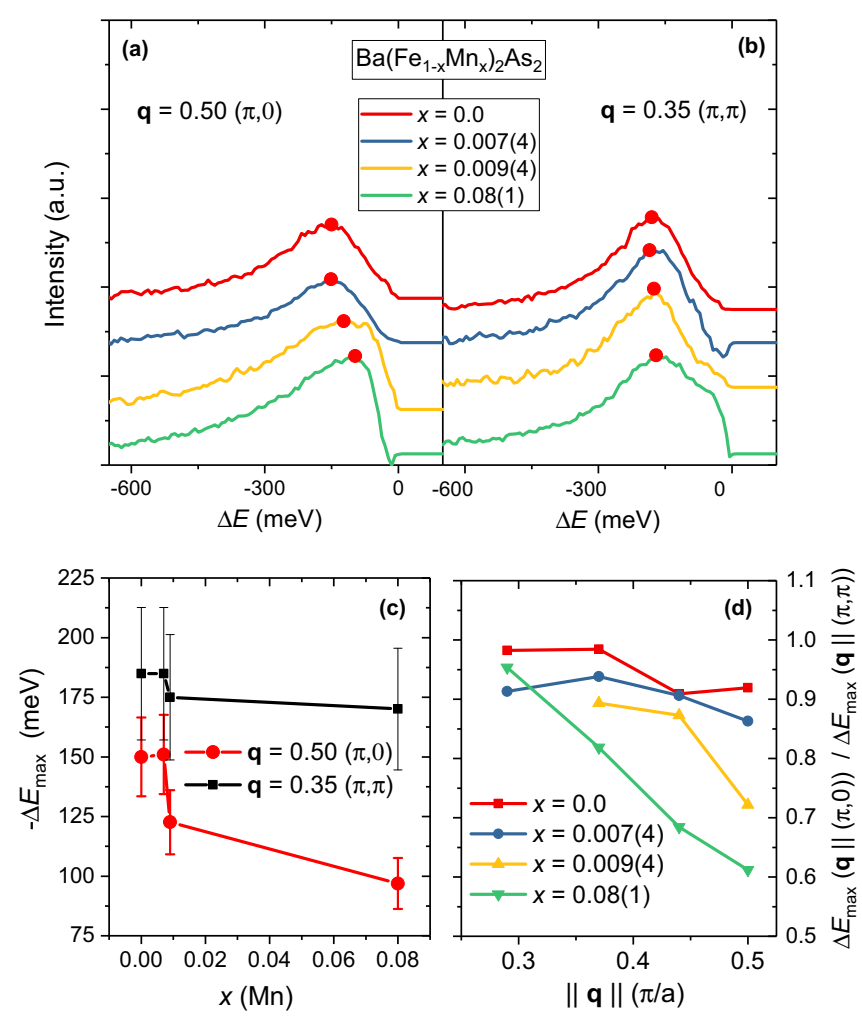

FIG. 5. RIXS spectra for maximum momentum transfer, with $q$ along the (a) $(\pi, 0)$ and (b) $(\pi, \pi)$ directions, for distinct $x$ (composition). The red dots mark the maximum of the excitations. (c) Energy loss, $-\Delta E_{\max }$, of the magnetic excitations as a function of $x$, for $\boldsymbol{q}$ along the $(\pi, 0)$ and $(\pi, \pi)$ directions. (d) Evolution of the anisotropy of the magnon modes as a function of momentum transfer $\|\boldsymbol{q}\|$, as expressed by the ratio of $\Delta E_{\max }(\boldsymbol{q})$ for $\boldsymbol{q}$ along the $(\pi, 0)$ and $(\pi, \pi)$ direction, for all investigated $x$.

of Mn impurities is investigated in Figs. 5(a)-5(d), where an overview of the composition dependence of RIXS spectra is presented. First we track in Figs. 5(a)-5(c) the doping evolution of the magnon modes for maximum momentum transfer. For all investigated samples, the modes along $(\pi, \pi)$ disperse to about $170 \mathrm{meV}$, while the modes along $(\pi, 0)$ present a clear trend toward softening starting from the $x=0.009$ sample [see also Fig. 5(c)]. A more comparative scenario is highlighted in Fig. 5(d). The anisotropy in the dispersion of the magnon modes as a function of $\|\boldsymbol{q}\|$ tends to disappear for small $\|\boldsymbol{q}\|$ and develops strongly with its increase, even for the $x=0.009$ sample. This result illustrates the impact of the cooperative behavior of magnetic disorder on the Fe-derived magnetic excitations due to the growing Mn impurity density that is related to an incipient Néel order.

\section{SUMMARY AND OUTLOOK}

Magnetic excitations in Mn substituted iron arsenides were investigated by means of RIXS at the Fe $L_{3}$ edge. The Fe derived excitations were shown to be composition and momentum dependent, in contrast to previous RIXS experiments of the arsenides. Our pnictide sample with the highest Mn doping $(x=0.08)$ displays magnetic excitations along the directions 
$(\pi, 0)$ and $(0, \pi)$ that are strongly damped and softened. It suggests that the absence of SC in phases of arsenides with Mn can thus be naturally explained by the lack of the $(\pi, 0)$ and $(0, \pi)$ fluctuations that promote the most efficient Cooper pairing channel. The cooperative behavior of $\mathrm{Mn}$ impurities was also highlighted. It was shown that small amounts of Mn have a large impact on the Fe derived excitations.

We propose that the intraorbital character of the $\mathrm{Fe}$ derived magnons is key to understand why the introduction of $Q_{\text {Néel }}=(\pi, \pi)$ fluctuations, derived from the Mn local spins, are able to scatter the magnons along the $(\pi, 0)$ and $(0, \pi)$ directions. Certainly, more theoretical and experimental work is needed to better understand this phenomenon. In this regard, the formal valence of $\mathrm{Mn}$ in $\mathrm{Ba}\left(\mathrm{Fe}_{1-x} \mathrm{Mn}_{x}\right)_{2} \mathrm{As}_{2}$ is $2+$, thus Mn impurities carry a large $S=5 / 2$ spin. It remains to be investigated if our results are due to the specifics of this spin configuration, or if it is a general feature concerning the presence of strongly localized moments at the FeAs layers. In this line of thinking, it is interesting to investigate phases containing $\mathrm{Cr}$, which do not present $\mathrm{SC}$ as well. The investigation of $\mathrm{Cu}$ containing samples is also invited. Although $\mathrm{Cu}$ leads to a SC phase [4] the critical temperature is low and that could be due to the scattering of $(\pi, 0) /(0, \pi)$ fluctua- tions. In this case, one expects $d$-wave $\mathrm{SC}$ in $\mathrm{Cu}$ containing samples.

\section{ACKNOWLEDGMENTS}

F.A.G. would like to acknowledge FAPESP (Grant No. 2016.22471-3) for financial support and the University of Zurich for financial support and hospitality. D.E.M. and T.S. acknowledge support by the Swiss National Science Foundation through the NCCR MARVEL. O.I., L.D., and J.C. acknowledges support from the Swiss National Science Foundation through the SINERGIA network Mott Physics beyond the Heisenberg Model and Grant No. BSSGI0_155873. L.D. is partially funded by a Swiss Goverment Ph.D. excellence scholarship. M.M.P., C.A., and P.G.P. acknowledge FAPESP (Grants No. 2015/15665-3, No. 2017/25269-3, and No. 2017/10581-1). C.M. acknowledges the support by the SNSF Grants No. PZ00P2_154867 and No. PP00P2_170597. The experiments were performed at the ADRESS beamline of the Swiss Light Source at the Paul Scherrer Institute. The authors acknowledge Rafael Fernandes for an illuminating discussion on the magnetic and electronic properties of the iron arsenides.
[1] Y. Kamihara, T. Watanabe, M. Hirano, and H. Hosono, J. Am. Chem. Soc. 130, 3296 (2008).

[2] K. Haule and G. Kotliar, New J. Phys. 11, 025021 (2009).

[3] D. C. Johnston, Adv. Phys. 59, 803 (2010).

[4] H. Hosono and K. Kuroki, Physica C (Amsterdam, Neth.) 514, 399 (2015).

[5] M. Rotter, M. Tegel, D. Johrendt, I. Schellenberg, W. Hermes, and R. Pöttgen, Phys. Rev. B 78, 020503(R) (2008).

[6] A. Thaler, H. Hodovanets, M. S. Torikachvili, S. Ran, A. Kracher, W. Straszheim, J. Q. Yan, E. Mun, and P. C. Canfield, Phys. Rev. B 84, 144528 (2011).

[7] P. F. S. Rosa, C. Adriano, T. M. Garitezi, M. M. Piva, K. Mydeen, T. Grant, Z. Fisk, M. Nicklas, R. R. Urbano, R. M. Fernandes, and P. G. Pagliuso, Sci. Rep. 4, 6252 (2014).

[8] Y. Texier, Y. Laplace, P. Mendels, J. T. Park, G. Friemel, D. L. Sun, D. S. Inosov, C. T. Lin, and J. Bobroff, Europhys. Lett. 99, 17002 (2012).

[9] S. Lafuerza, H. Gretarsson, F. Hardy, T. Wolf, C. Meingast, G. Giovannetti, M. Capone, A. S. Sefat, Y.-J. Kim, P. Glatzel, and L. de' Medici, Phys. Rev. B 96, 045133 (2017).

[10] J. Pelliciari, Y. Huang, K. Ishii, C. Zhang, P. Dai, G. F. Chen, L. Xing, X. Wang, C. Jin, H. Ding, P. Werner, and T. Schmitt, Sci. Rep. 7, 8003 (2017).

[11] S. Jiang, H. Xing, G. Xuan, Z. Ren, C. Wang, Z.-a. Xu, and G. Cao, Phys. Rev. B 80, 184514 (2009).

[12] M. Matusiak, Z. Bukowski, and J. Karpinski, Phys. Rev. B 83, 224505 (2011).

[13] M. M. Korshunov and I. Eremin, Phys. Rev. B 78, 140509 (2008).

[14] J. Zhang, R. Sknepnek, R. M. Fernandes, and J. Schmalian, Phys. Rev. B 79, 220502 (2009).

[15] R. M. Fernandes and A. J. Millis, Phys. Rev. Lett. 110, 117004 (2013).
[16] M. G. Kim, A. Kreyssig, A. Thaler, D. K. Pratt, W. Tian, J. L. Zarestky, M. A. Green, S. L. Bud'ko, P. C. Canfield, R. J. McQueeney, and A. I. Goldman, Phys. Rev. B 82, 220503 (2010).

[17] G. S. Tucker, D. K. Pratt, M. G. Kim, S. Ran, A. Thaler, G. E. Granroth, K. Marty, W. Tian, J. L. Zarestky, M. D. Lumsden, S. L. Bud'ko, P. C. Canfield, A. Kreyssig, A. I. Goldman, and R. J. McQueeney, Phys. Rev. B 86, 020503 (2012).

[18] D. S. Inosov, G. Friemel, J. T. Park, A. C. Walters, Y. Texier, Y. Laplace, J. Bobroff, V. Hinkov, D. L. Sun, Y. Liu, R. Khasanov, K. Sedlak, P. Bourges, Y. Sidis, A. Ivanov, C. T. Lin, T. Keller, and B. Keimer, Phys. Rev. B 87, 224425 (2013).

[19] H. Suzuki, T. Yoshida, S. Ideta, G. Shibata, K. Ishigami, T. Kadono, A. Fujimori, M. Hashimoto, D. H. Lu, Z.-X. Shen, K. Ono, E. Sakai, H. Kumigashira, M. Matsuo, and T. Sasagawa, Phys. Rev. B 88, 100501 (2013).

[20] G. Ghiringhelli, A. Piazzalunga, C. Dallera, G. Trezzi, L. Braicovich, T. Schmitt, V. N. Strocov, R. Betemps, L. Patthey, X. Wang, and M. Grioni, Rev. Sci. Instrum. 77, 113108 (2006).

[21] V. N. Strocov, T. Schmitt, U. Flechsig, T. Schmidt, A. Imhof, Q. Chen, J. Raabe, R. Betemps, D. Zimoch, J. Krempasky, X. Wang, M. Grioni, A. Piazzalunga, and L. Patthey, J. Synchrotron Radiat. 17, 631 (2010).

[22] J. T. Park, D. S. Inosov, A. Yaresko, S. Graser, D. L. Sun, P. Bourges, Y. Sidis, Y. Li, J.-H. Kim, D. Haug, A. Ivanov, K. Hradil, A. Schneidewind, P. Link, E. Faulhaber, I. Glavatskyy, C. T. Lin, B. Keimer, and V. Hinkov, Phys. Rev. B 82, 134503 (2010).

[23] T. M. Garitezi, C. Adriano, P. F. S. Rosa, E. M. Bittar, L. Bufaiçal, R. L. d. Almeida, E. Granado, T. Grant, Z. Fisk, M. A. Avila, R. A. Ribeiro, P. L. Kuhns, A. P. Reyes, R. R. Urbano, and P. G. Pagliuso, Braz. J. Phys. 43, 223 (2013).

[24] W. L. Yang, A. P. Sorini, C.-C. Chen, B. Moritz, W.-S. Lee, F. Vernay, P. Olalde-Velasco, J. D. Denlinger, B. Delley, J.-H. 
Chu, J. G. Analytis, I. R. Fisher, Z. A. Ren, J. Yang, W. Lu, Z. X. Zhao, J. van den Brink, Z. Hussain, Z.-X. Shen, and T. P. Devereaux, Phys. Rev. B 80, 014508 (2009).

[25] K.-J. Zhou, Y.-B. Huang, C. Monney, X. Dai, V. N. Strocov, N.-L. Wang, Z.-G. Chen, C. Zhang, P. Dai, L. Patthey, J. van den Brink, H. Ding, and T. Schmitt, Nat. Commun. 4, 1470 (2013).

[26] J. Pelliciari, Y. Huang, T. Das, M. Dantz, V. Bisogni, P. O. Velasco, V. N. Strocov, L. Xing, X. Wang, C. Jin, and T. Schmitt, Phys. Rev. B 93, 134515 (2016).

[27] J. Pelliciari, K. Ishii, M. Dantz, X. Lu, D. E. McNally, V. N. Strocov, L. Xing, X. Wang, C. Jin, H. S. Jeevan, P. Gegenwart, and T. Schmitt, Phys. Rev. B 95, 115152 (2017).

[28] J. Pelliciari, M. Dantz, Y. Huang, V. Strocov, L. Xing, X. Wang, C. Jin, and T. Schmitt, Appl. Phys. Lett. 109, 122601 (2016).

[29] H. Suzuki, M. Minola, Y. Lu, Y. Peng, R. Fumagalli, E. Lefrançois, T. Loew, J. Porras, K. Kummer, D. Betto, S. Ishida, H. Eisaki, C. Hu, X. Zhou, M. W. Haverkort, N. B. Brookes, L. Braicovich, G. Ghiringhelli, M. L. Tacon, and B. Keimer, npj Quantum Materials 3, 65 (2018).

[30] One must keep in mind that the line broadening usually shifts the magnon excitation frequency to lower energies in comparison with the peak position. Indeed in the damped harmonic oscillator model $\omega_{q}=\sqrt{\omega_{0}^{2}-(\Gamma / 2)^{2}}$ where $\omega_{0}$ is the bare frequency, closely corresponding to the peak position and $\Gamma$ relates to the half maximum linewidth.

[31] M. Le Tacon, G. Ghiringhelli, J. Chaloupka, M. Moretti Sala, V. Hinkov, M. Haverkort, M. Minola, M. Bakr, K. Zhou,
S. Blanco-Canosa, C. Monney, Y. Song, G. Sun, C. Lin, G. De Luca, M. Salluzzo, G. Khaliullin, T. Schmitt, L. Braicovich, and B. Keimer, Nat. Phys. 7, 725 (2011).

[32] M. P. M. Dean, G. Dellea, R. Springell, F. Yakhou-Harris, K. Kummer, N. Brookes, X. Liu, Y.-J. Sun, J. Strle, T. Schmitt, L. Braicovich, G. Ghiringhelli, I. Bozovic, and J. Hill, Nat. Mater. 12, 1019 (2013).

[33] C. Monney, K. J. Zhou, H. Cercellier, Z. Vydrova, M. G. Garnier, G. Monney, V. N. Strocov, H. Berger, H. Beck, T. Schmitt, and P. Aebi, Phys. Rev. Lett. 109, 047401 (2012).

[34] V. Bisogni, S. Catalano, R. J. Green, M. Gibert, R. Scherwitzl, Y. Huang, V. N. Strocov, P. Zubko, S. Balandeh, J.-M. Triscone, G. Sawatzky, and T. Schmitt, Nat. Commun. 7, 13017 (2016).

[35] M. Kanász-Nagy, Y. Shi, I. Klich, and E. A. Demler, Phys. Rev. B 94, 165127 (2016).

[36] M. Minola, Y. Lu, Y. Y. Peng, G. Dellea, H. Gretarsson, M. W. Haverkort, Y. Ding, X. Sun, X. J. Zhou, D. C. Peets, L. Chauviere, P. Dosanjh, D. A. Bonn, R. Liang, A. Damascelli, M. Dantz, X. Lu, T. Schmitt, L. Braicovich, G. Ghiringhelli, B. Keimer, and M. Le Tacon, Phys. Rev. Lett. 119, 097001 (2017).

[37] E. Kaneshita, K. Tsutsui, and T. Tohyama, Phys. Rev. B 84, 020511 (2011).

[38] S. Graser, T. A. Maier, P. J. Hirschfeld, and D. J. Scalapino, New J. Phys. 11, 025016 (2009).

[39] M. N. Gastiasoro and B. M. Andersen, Phys. Rev. Lett. 113, 067002 (2014).

[40] X. Wang and R. M. Fernandes, Phys. Rev. B 89, 144502 (2014). 